\title{
Características do uso de fármacos sem prescrição por graduandos em Enfermagem
}

\section{Characteristics of drug use without prescription in undergraduate Nursing students}

\author{
1 Cristiane Gorgati Guidoreni crisguidoreni@gmail.com \\ 2 Mariana Emília da Silveira Bittencourt \\ ${ }^{3}$ Naiara de Almeida Pires
}

\footnotetext{
1 Docente dos cursos de Medicina, Nutrição e Enfermagem do Centro Universitário de Volta Redonda, UniFOA.

2 Docente do curso de Enfermagem do Centro Universitário de Volta Redonda, UniFOA.

3 Discente do curso de Enfermagem do Centro Universitário de Volta Redonda, UniFOA.
}

\section{RESUMO}

Automedicação é a iniciativa de selecionar e utilizar medicamentos para tratar sintomas e patologias autodiagnosticadas, ato que deve ser entendido como um dos elementos do autocuidado. Objetivamos abordar a automedicação com acadêmicos de enfermagem, e avaliar a incidência e características dessa prática. Como metodologia, adotamos a pesquisa de campo, descritiva e exploratória, com abordagem quantitativa, e como instrumento para a coleta de dados utilizamos um questionário aplicado aos graduandos do $1^{\circ}$ ao $10^{\circ}$ períodos do curso de enfermagem do Centro Universitário de Volta Redonda - UniFOA. Foram analisados os dados de 155 alunos, em que 141 confirmaram se automedicarem, 90,32\% acreditam que essa ação mascara sintomas de doenças e, dos mais variados fármacos, os Antiinflamatórios não esteroides obtiveram maior índice de uso. Concluímos que é de grande preocupação a taxa elevada de graduandos que se automedicam e deve ser reforçada em universidades a importância do conhecimento e consciência dos riscos que essa prática oferece.

\section{PALAVRAS-CHAVE}

Farmacoepidemiologia, automedicação, enfermagem, graduação.

\begin{abstract}
self-medication is the initiative to select and use drugs to treat symptoms and self-diagnose pathologies, act that must be understood as one of self-care elements. We aim to address selfmedication with nursing students, and assess the incidence and characteristics of this practice. The methodology adopted was field research, descriptive and exploratory, with a quantitative approach, and as a tool for data collection we used a questionnaire to be answered by undergraduate students from 1st to 10th periods of the nursing program of the Centro Universitário de Volta Redonda - UniFOA. Data from 155 students were analyzed, where 141 confirmed self-medicating, $90.32 \%$ believe that this action masks symptoms of disease, and Nonsteroidal anti-inflammatory drug had higher use rate. We conclude that the high rate of graduates who selfmedicate is reason for great concern and universities should strengthen the importance of knowledge and awareness of the risks that this practice offers.
\end{abstract}

\section{KEYWORDS}

Pharmacoepidemiology, self-medication, nursing, graduation.

\section{Como você deve citar?}

GUIDORENI, Cristiane Gorgati; BITTENCOURT, Mariana Emília da Silveira; PIRES, Naiara de Almeida. Características do uso de fármacos sem prescrição por graduandos em Enfermagem. Cadernos UniFOA, Volta Redonda, n. 29, p. 129-136, dez. 2015. 


\section{INTRODUÇÃO}

A automedicação é a iniciativa que as pessoas têm de selecionar e utilizar medicamentos para tratar sintomas e patologias autodiagnosticadas, ato que deve ser entendido como um dos elementos do autocuidado. Define-se como automedicação responsável a prática pela qual os indivíduos tratam de seus sintomas e complicações de saúde com medicamentos aprovados e disponíveis para serem adquiridos como indicado (SANTOS, 2012 apud OMS, 2003).

0 uso indiscriminado de medicamentos tem como consequência efeitos indesejáveis, complicações, enfermidades e iatrogenias, mascaramento de doenças evolutivas, representando portando problemas a serem prevenidos. 0 risco dessa prática evidentemente se correlaciona com o grau de instrução e informação do usuário sobre medicamentos. A qualidade da oferta de medicamentos e a eficiência do trabalho dos que controlam de várias maneiras esse mercado também exercem papel de grande relevância nos riscos implícitos na automedicação (ARRAIAS e cols,1997).

Esses problemas podem ser amenizados se a equipe multidisciplinar da saúde estiver apta a compartilhar deste conhecimento com os indivíduos que não têm a profunda informação das consequências adquiridas com esse ato, assim ressaltando a importância do enfermeiro estar preparado para realizar a educação em saúde onde quer que se encontre.

A dificuldade ao acesso aos serviços de saúde pode ocasionar uma procura por formas alternativas de tratamento de determinados sintomas momentaneamente, ampliando a incidência da automedicação e contribuindo para o aumento da crise do Sistema Único de Saúde, fato que contribui para que novos estudos sobre automedicação sejam realizados atualmente (ASCARI, e cols., 2014).

Vários trabalhos demonstram que esta prática é muito comum (DAMASCENO e cols., 2007; MUSSOLIN e cols., 2004; PENNA e cols., 2011; NARLOCH e cols., 2004), em idosos pode chegar a 80\% (CASCAES e cols., 2008) e na população em geral a 46\% (LOYOLA e cols, 2002). Em trabalhos realizados com estudantes estes valores foram superiores a 70\% (DAMASCENO e cols., 2007; PENNA e cols., 2011). Mesmo que muitas vezes esta prática possa ter resultados favoráveis como melhoria de sintomas ou resolução do problema de saúde, outras vezes pode trazer prejuízos à saúde do indivíduo, como mascaramento dos problemas de saúde, intoxicação, reações adversas, interações medicamentosas, desenvolvimento de resistência entre outros (ASSOCIAÇÃO MÉDICA BRASILEIRA, 2001; OGAWA e cols., 2011).

Segundo Tomasi (2007), o risco dessa prática está correlacionado com o grau de instrução e informação dos usuários sobre medicamentos, bem como com a acessibilidade dos mesmos ao sistema de saúde.

Dessa forma, traçamos como objetivos específicos identificar o número de alunos graduandos de enfermagem que utilizam a automedicação e as características dessa prática.

\section{MÉTODOS E TÉCNICAS}

Trata-se de um estudo descritivo e exploratório, com abordagem quantitativa, realizado no Centro Universitário de Volta Redonda - UniFOA entre os acadêmicos de enfermagem no primeiro semestre de 2015.

O instrumento de coleta de dados utilizado foi um questionário composto por 9 questões sobre a prática da automedicação em sua rotina. 
O projeto foi submetido e aprovado pelo Comitê de Ética e Pesquisa do UniFOA (CAAE: 44519715.0.0000.5237). A pesquisa foi desenvolvida mediante esclarecimentos gerais aos participantes e após estes assinarem o termo de Consentimento Livre e Esclarecido, de acordo com a resolução 196/96 do Conselho Nacional de Saúde.

Foram utilizados como critério de inclusão os discentes do curso de enfermagem do Centro Universitário de Volta Redonda-RJ, que aceitaram participar do estudo assinando o Termo de Consentimento Livre e Esclarecido - TCLE e que estivessem cursando entre o $1^{\circ}$ ao $10^{\circ}$ período do curso de graduação em Enfermagem. A coleta de dados ocorreu no mês de maio de 2015.

A análise estatística dos dados foi realizada com o auxílio do software Excel para a tabulação e construção dos gráficos.

\section{RESULTADOS E DISCUSSÃO}

Ao todo, $155(67,10 \%)$ dos 231 graduandos matriculados no curso de enfermagem participaram da pesquisa. 0 período com maior participação foi o $3^{\circ}$, com $84,38 \%$ da turma, e o com menor participação foi o $7^{\circ}$, onde apenas $36,00 \%$ dos alunos participaram (tabela 1).

Tabela 1 - Porcentagem de alunos participantes da pesquisa.

\begin{tabular}{cccc}
\hline Período & $\begin{array}{c}\text { Total de alunos do } \\
\text { período }\end{array}$ & Alunos participantes & \% do total \\
\hline 1 & 57 & 44 & $77,19 \%$ \\
\hline 3 & 32 & 27 & $84,38 \%$ \\
\hline 4 & 21 & 10 & $47,62 \%$ \\
\hline 5 & 18 & 14 & $77,78 \%$ \\
\hline 6 & 11 & 8 & $72,73 \%$ \\
\hline 7 & 25 & 9 & $36,00 \%$ \\
\hline 8 & 17 & 12 & $70,59 \%$ \\
\hline 9 & 27 & 14 & $51,85 \%$ \\
\hline 10 & 23 & 17 & $73,91 \%$ \\
\hline
\end{tabular}

Fonte: Questionário semiestruturado.

A maioria da amostra era do sexo feminino (85,4\%), sendo que $1,29 \%$ (2 pessoas) não responderam quanto ao sexo.

Apenas 8,39\% dos alunos afırmaram não ter feito uso de medicação sem prescrição médica, 1 aluno não respondeu a esta questão e 141 dos 155 graduandos disseram já ter utilizado fármacos sem prescrição (Figura 1).

Diversos estudos mostram a alta taxa de automedicação entre estudantes de enfermagem, variando entre $72,0 \%$ e 91,2\% (Santos B. e cols, 2012; Holthausen CN e cols, 2001; Souza LAF e cols, 2011; Silva FM e cols, 2014; Lopes, W. F. L. e cols, 2014). Nossos resultados demonstram que os alunos da graduação em enfermagem do Centro Universitário de Volta Redonda, encontram-se dentro dessa faixa, com 90,96\% de incidência de automedicação. 
Santos B. e colaboradores (2012) relataram encontrar discrepâncias entre a proporção de alunos que afırmaram fazer uso de automedicação e aqueles que citaram fármacos utilizados sem prescrição médica. Tal resultado também foi encontrado entre nossos alunos, enquanto $90,96 \%$ dos alunos afirmaram já ter feito uso de automedicação, $97,41 \%$ de todos os alunos pesquisados citaram ao menos um fármaco de que já fizeram uso sem prescrição médica. Dos 13 alunos que afirmaram não ter praticado automedicação, 9 entraram em contradição, citando fármacos utilizados.

Os resultados demonstraram também que a grande maioria desses alunos $(90,32 \%)$ acredita que o uso desses fármacos pode mascarar os sintomas da doença. Tal resultado demonstra que o conhecimento sobre alguns dos malefícios da automedicação não parece inibir a prática, uma vez que mesmo tendo declarado conhecer este efeito, os alunos fazem uso de medicamentos sem prescrição médica.

Quanto ao tempo de uso dos fármacos, 23,23\% dos alunos disseram utilizá-los em média por 1 dia, $20,0 \%$ por até dois dias e $40,0 \%$ por 3 a 5 dias (Figura 2). Vale destacar que dentre os alunos que afirmaram utilizar tais fármacos por mais que 5 dias, 2 disseram fazer uso dos mesmos todos os dias, e 1, por meses.

Figura 1 - Porcentagem de alunos que afirmaram já ter feito uso de fármacos sem prescrição médica.

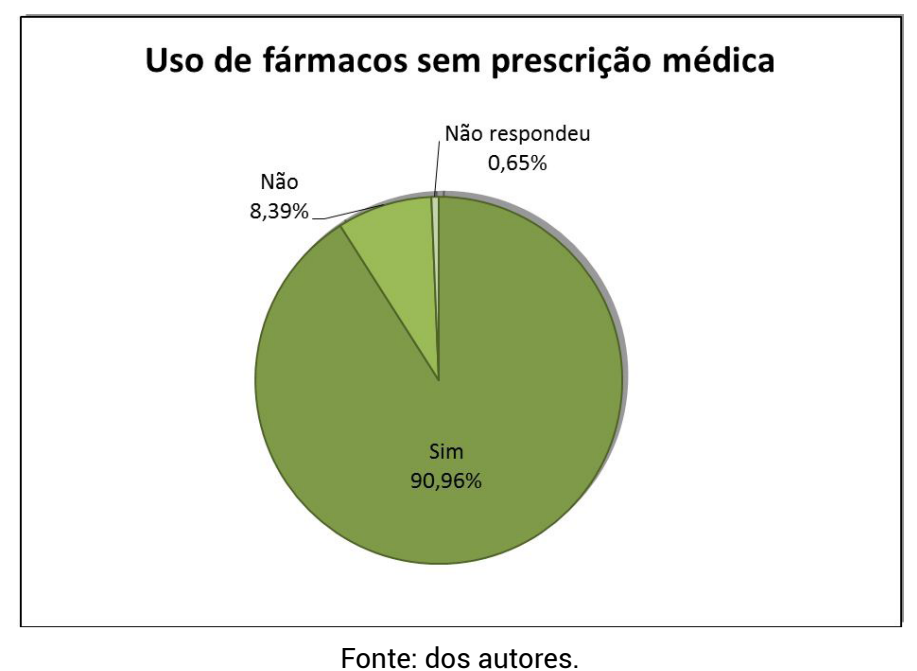

Figura 2 - Porcentagem de alunos segundo o tempo médio de uso dos fármacos.

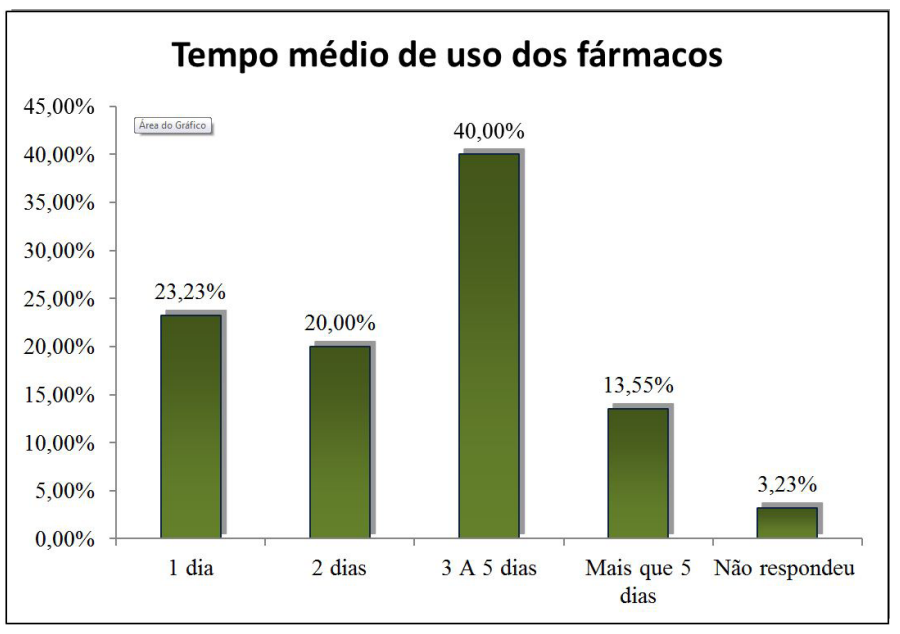

Fonte: dos autores. 
A pesquisa buscou avaliar também quais os principais fármacos utilizados sem prescrição médica pelos alunos. A figura 3 mostra que dentre os fármacos mais utilizados, os anti-inflamatórios não esteroides (AINES) são os mais presentes: $96,1 \%$ dos alunos afirmaram já ter feito uso de dipirona, $83,9 \%$ utilizaram paracetamol, 38,7\% disseram já ter usado ácido acetil salicílico e 37,4\% diclofenaco ácido sem prescrição. Dentre os demais fármacos citados, o cloridrato de nafazolina ou outros descongestionantes nasais somaram $60,0 \%$ dos alunos. Mais da metade dos alunos afirmaram já ter usado inibidores da bomba de prótons $(51,6 \%)$ e dimenidrinato $(51,0 \%)$.

Figura 3 - Fármacos utilizados sem prescrição médica.

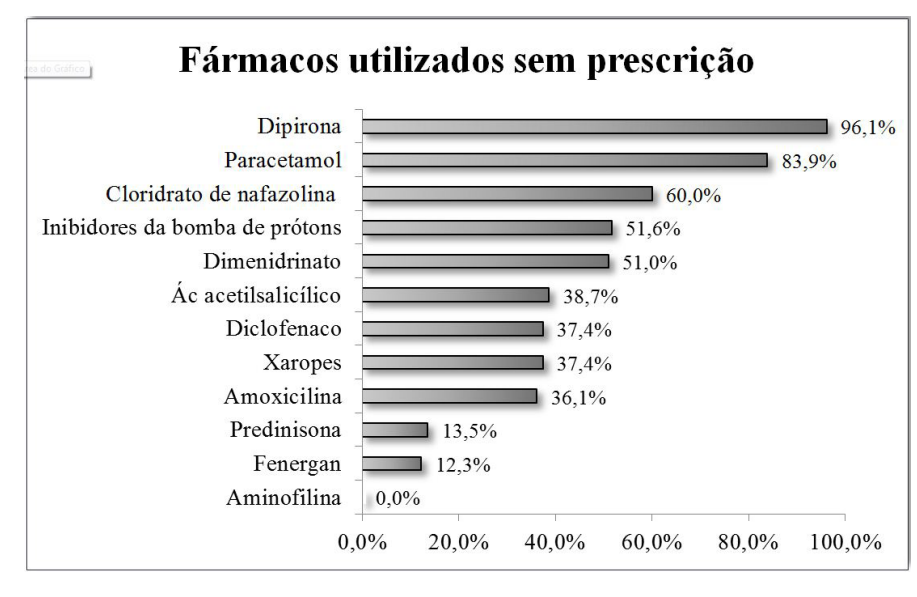

Fonte: dos autores.

Quando juntamos os alunos que responderam já ter feito uso de algum dos AINES, vimos que apenas 4 alunos afirmaram não ter utilizado esses fármacos, ou seja, $96,12 \%$ dos graduandos já fizeram uso de AINES sem prescrição médica. Outros dados que chamam a atenção são a incidência do uso de inibidores da bomba de prótons $(51,61 \%)$ e o fato de que mais de um terço dos graduandos $(36,1 \%)$ já fizeram uso de amoxicilina sem prescrição médica. Dentre os sintomas que levaram os graduandos a fazer uso desses medicamentos, os mais citados foram a dor de cabeça $(91,6 \%)$, resfriado $(73,5 \%)$, febre $(69,7 \%)$ e dor muscular (63,2\%) (Figura 4).

Figura 4 - Sintomas citados como causa da busca pela automedicação.

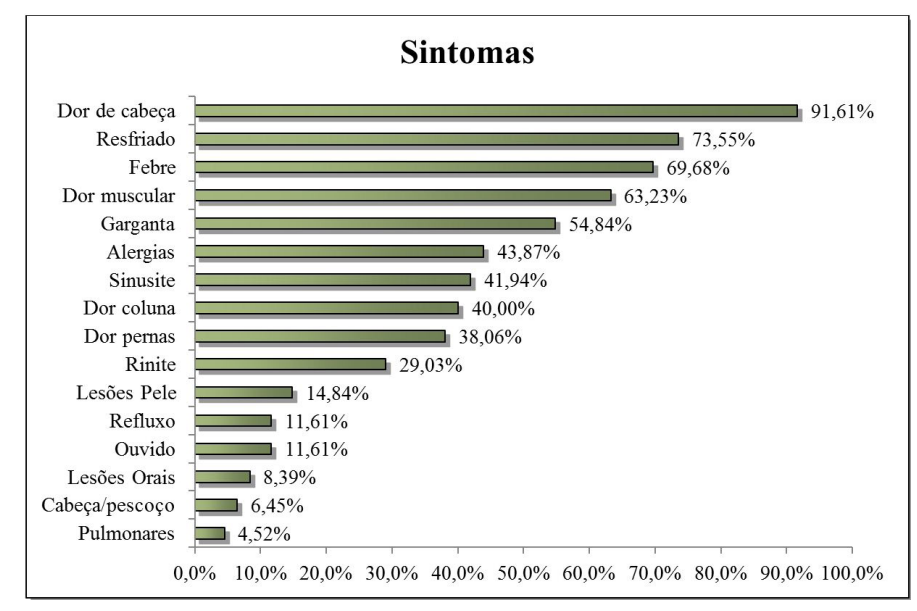

Fonte: dos autores. 
Quando questionados sobre os possíveis motivos pelo qual fizeram uso de medicamentos sem prescrição, a razão mais citada foi a busca do alívio rápido dos sintomas (90,32\%). Causas como a dificuldade de acesso ao serviço público de saúde ou mesmo ao particular foram pouco frequentes, $12,26 \%$ e 2,58\% respectivamente. A segunda causa mais citada foi o conhecimento sobre a patologia, com $40,0 \%$ dos graduandos (Figura 5). Confirmando os resultados obtidos no presente trabalho, alguns estudos mostram que o tratamento da cefaleia é feito principalmente com analgésicos e que a automedicação é bastante comum nestes casos. (OLIVEIRA \& PELÓGIA, 2011).

Durante a faculdade, o graduando passa por situações de estresse e sobrecarga que podem acarretar em dores que justificariam o alto consumo de analgésicos, anti-inflamatórios e miorrelaxantes, porém estudos demonstram que estes medicamentos são muito consumidos, não só em casos de estresse ou cansativa jornada de aulas. É sabido que as classes terapêuticas mais utilizadas em crianças, por exemplo, são antiinflamatórios não esteroidais, analgésicos e antibióticos (PFAFFENBACH, 2010; MEDEIROS, PEREIRA, MEDEIROS, 2011).

0 alto consumo de analgésicos, devido à cefaleia, gripes, entre outros, pode levar a efeitos adversos tais como desconforto gástrico, úlceras e gastrites (GELLER et. al, 2012; UFGRS, 2002).

Figura 5 - Motivos pelos quais os graduandos utilizam medicamentos sem prescrição médica.

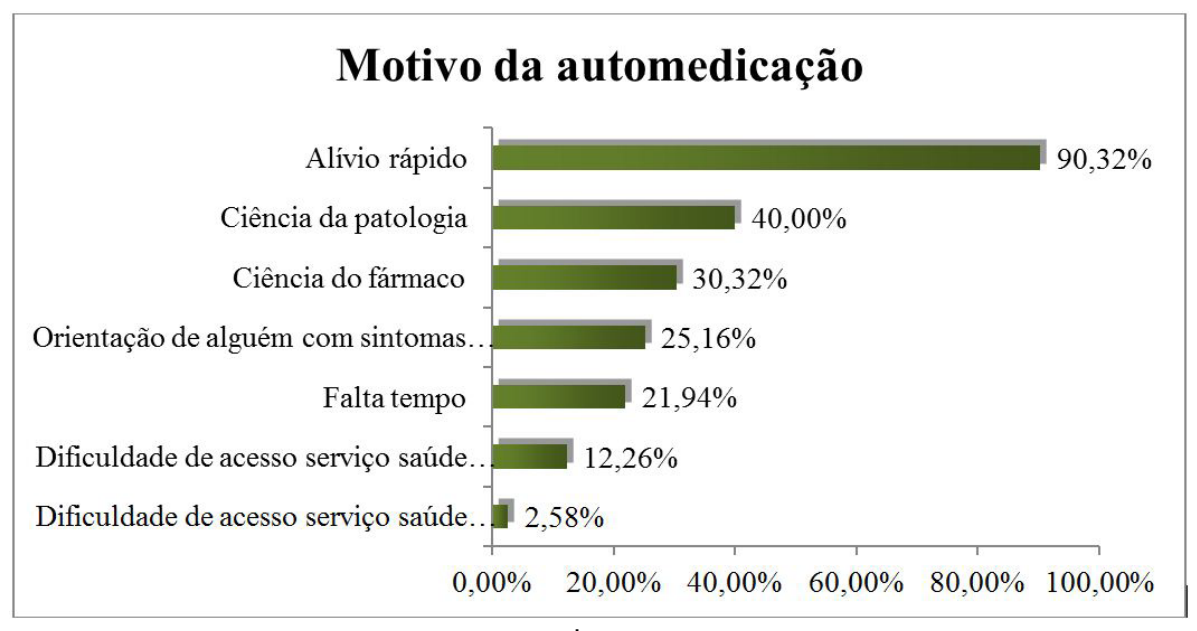

Fonte: dos autores.

Apesar disso, quando foi questionado a esses alunos se já tinham tido reações adversas ao uso desses medicamentos, apenas $12,26 \%$ afirmaram já ter tido algum tipo de reação adversa. A baixa ocorrência desses efeitos adversos pode contribuir para a alta taxa de automedicação, uma vez que os alunos se sentem ilusoriamente seguros com o uso.

\section{CONCLUSÃO}

Com os dados adquiridos com essa pesquisa podemos concluir que $90,97 \%$, dos graduandos afirmaram já ter feito uso de fármacos sem prescrição. Os resultados foram bem elevados, o que é preocupante, uma vez que se trata de graduandos de enfermagem, profissionais que devem visar à segurança do paciente, o que se aplica integralmente à atuação destes no que se refere à terapêutica medicamentosa. Outro resultado que nos leva a refletir sobre o nível de conhecimento dos graduandos é o de apenas quatro discentes afırmarem não ter utilizado AINES sem prescrição, uma vez que esses fármacos agem como inibidores da síntese de prostaglandinas causando diversos prejuízos a saúde como gastrites e problemas na coagulação sanguínea (GELLER e cols, 2012). 


\section{REFERÊNCIAS}

ARRAIS, P. S. D. et al. Perfil da automedicação no Brasil. Rev. saúde pública, v. 31, n. 1, p. 71-7, 1997.

ASCARI, R. A. et al. "Estratégia saúde da família: automedicação entre os usuários." UNINGÁ Review 18.2 (2014).Associação Médica Brasileira. Automedicação. RevAssocMedBras ; 47(4):269-270; 2001.

CASCAES, A. E.; FALCHETTI, M. L.; GALATO, D. Perfil da automedicação em idosos participantes de grupos da terceira idade de uma cidade do sul do Brasil. Arq Cat Med ; 37(1):63-39; 2008.

CASTRO, L. L. C. Pharmacoepidemiology in Brazil: evolution and prospects. Ciência \& Saúde Coletiva, v. 4, n. 2, p. 405-410, 1999.

DAMASCENO, D. D.et al.Automedicação entre graduandos de enfermagem, farmácia e odontologia da Universidade Federal de Alfenas. Reme Rev Min Enferm ; 11(1):48-52; 2007.

GALATO, D.; MADALENA, J.; PEREIRA, G. B.;Automedicação em estudantes universitários: a influência da área de formação. Ciênc. saúde coletiva[online]., vol.17, n.12, pp. 3323-3330. ISSN 1413-8123. http:// dx.doi.org/10.1590/S1413-81232012001200017; 2012.

GELLER, M.; KRYMCHANTOWSKI, A. V.; STEINBRUCH, M.; CUNHA, K. S.; RIBEIRO, M. G.; OLIVEIRA, L.; OZERI, D.; DAHER, J. P. L. Utilização do diclofenaco na prática clínica: revisão das evidências terapêuticas e ações farmacológicas. RevBrasClin Med., São Paulo, v.10, n. 1, pg. 29-38, jan./fev., 2012.

HOLTHAUSEN C.N., ALDEMAN J., BORGES J.N., MATTAR S.B., MIGUEL M.D. Automedicação e os acadêmicos da área da saúde. Infarma.; 13(1/2):74-5; 2001.

LOYOLA, F. A.; et al. Prevalência e fatores associados à automedicação: resultados do projeto Bambuí. RevSaudePublica ; 36(1):55-62; 2002.

MARIN, N.; et al. Assistência farmacêutica para gerentes municipais. In: Assistência farmacêutica para gerentes municipais. Organização Pan-Americana da Saúde, 2003.

MEDEIROS, R. A.; PEREIRA, V. G.; MEDEIROS, S.M. Vigilância em saúde na enfermagem: o caso das medicações sem prescrição em crianças. Esc. Anna Nery, R

io de Janeiro, v.15 n. 2 Abr./Jun., 2011.

MUSSOLIN, N. M.; $\boldsymbol{A}$ automedicação: um estudo entre universitários de enfermagem e de relações públicas [dissertação]. São Paulo (SP): Secretaria da Saúde. Coordenação dos Institutos de Pesquisa. Programa de Pós-Graduação em Ciências; 2004.

NARLOCH, L.; et al.Automedicação entre os acadêmicos da UFSC. In: $4^{\text {a }}$ Semana de Ensino, Pesquisa e Extensão [anais na internet] Set 22-25; Florianópolis, Brasil; 2004.

OGAWA, A.; et al. Estudo comparativo sobre automedicação em estudantes do segundo ano de enfermagem e medicina e moradores do bairro Vila Nova. Espaço Saude [periódico na internet]. [acessado 2011 jul 18]; 3(2):[13 p];2001 
OLIVEIRA, A. L. M.; PELÓGIA, N. C. C. Cefaleia como principal causa de automedicação entre os profissionais da saúde não prescritores. Rev. Dor, São Paulo, v.12, n. 2 Abr./Jun., 2011.

PENNA, A.B.; et al. Análise da Prática da Automedicação em Universitários do Campus Magnus - Unipac - Barbacena, MG. In: Anais do $2^{\circ}$ Congresso Brasileiro de Extensão Universitária[anais na internet] Set 12-15; Belo Horizonte, Brasil [acessado 2011 jul 18]; 2004

PFAFFNBACH, G. Automedicação em crianças: um problema de saúde pública. Rev. paul. pediatr., São Paulo, v. 28, n. 3, Set., 2010.

SANTOS, B. et al. Incidência da Automedicação em Graduandos de Enfermagem. J healthSci Inst;;30(2):156$60 ; 2012$.

SILVA, F.M.; GOULART, F.C.; LAZARINI, C. Caracterização da prática de automedicação e fatores associados entre universitários do curso de Enfermagem. Revista Eletrônica de Enfermagem, [S.I.], v. 16, n. 3, p. 644-51, set. 2014.

SOUZA L.A.F., SILVA C.D., FERRAZ G.C., FALEIROS SOUSA F.A.E., PEREIRA L.V. Prevalência e caracterização da prática de automedicação para alívio da dor entre estudantes universitários de enfermagem. Rev. Latino-Am. Enfermagem 19(2):[07 telas] mar-abr 2011.

TOMASI, E. et al.Condições de trabalho e automedicação em profissionais da rede básica de saúde da zona urbana de Pelotas, RS.RevBrasEpidemiol; 10(1):66-74;2007 